\title{
Clinical implications of the MDM2 SNP309 and p53 Arg72Pro polymorphisms in transitional cell carcinoma of the bladder
}

\author{
YOHEI HORIKAWA ${ }^{1}$, JUNICHI NADAOKA ${ }^{1}$, MITSURU SAITO ${ }^{1}$, \\ TERUAKI KUMAZAWA ${ }^{1}$, TAKAMITU INOUE ${ }^{1}$, TAKESHI YUASA ${ }^{1}$, NORIHIKO TSUCHIYA ${ }^{1}$, \\ HIROYUKI NISHIYAMA ${ }^{2}$, OSAMU OGAWA ${ }^{2}$ and TOMONORI HABUCHI ${ }^{1}$ \\ ${ }^{1}$ Department of Urology, Akita University School of Medicine, Akita; \\ ${ }^{2}$ Department of Urology, Kyoto University Graduate School of Medicine, Kyoto, Japan
}

Received February 13, 2008; Accepted March 24, 2008

\begin{abstract}
Recent studies have shown that functional polymorphisms at the MDM2 SNP309 T/G and p53 Arg72Pro may be associated with cancer susceptibility. However, the role of these polymorphisms on the risk of transitional cell carcinoma of the bladder (TCCB) and clinical outcome remains unknown. SNP309 and p53 Arg72Pro polymorphisms were genotyped in 227 patients and 266 control subjects. The association between each polymorphism, TCCB risk and clinical outcome was evaluated by using a logistic regression model, a Kaplan-Meier curve with the log-rank test, or a Cox proportional hazard model. No significant associations between the polymorphisms and TCCB risk were found. On the MDM2 SNP309, the TT patients with superficial TCCB tended to have a longer recurrence-free survival than the TG or GG patients after transurethral resection $(\mathrm{P}=0.074)$. On the $p 53$ Arg72Pro, the Pro/Pro patients with superficial TCCB had a significantly lower risk for recurrence than the Arg/Pro or Arg/Arg patients [Hazard ratio (HR), 0.364; 95\% confidence interval (CI), 0.14-0.93]. In contrast, the Pro/Pro patients following radical cystectomy showed a significantly poorer survival and a higher risk of disease-specific death than the Arg/Pro + Arg/Arg patients (HR, 2.76; 95\% CI, 1.11-6.84). MDM2 SNP309 and p53 Arg72Pro polymorphisms might influence the clinical outcome of TCCB in a distinctive way between superficial TCCB and invasive TCCB. The results may reflect marked differences in the genetic background between superficial and an invasive type of TCCB.
\end{abstract}

Correspondence to: Dr Tomonori Habuchi, Department of Urology, Akita University School of Medicine, 1-1-1 Hondo, Akita 010-8543, Japan

E-mail: thabuchi@doc.med.akita-u.ac.jp

Abbreviations: SNP, single nucleotide polymorphism; TCCB, transitional cell carcinoma of the bladder; DSS, disease-specific survival; RFS, recurrence-free survival

Key words: bladder cancer, MDM2 SNP309, p53 Arg72Pro, polymorphism, prognosis

\section{Introduction}

The p53 tumor suppressor gene is a key molecule in human malignancies and the dysfunction of the p53 pathway has been shown to contribute to the initiation and progression of a tumor $(1,2)$. A functional polymorphism at p53 Arg72Pro has been extensively studied and the $\operatorname{Arg} 72$ variant has been shown to be a stronger and faster inducer of apoptosis than the Pro72 variant (3-6). Although many researchers have investigated the association between $p 53$ Arg72Pro polymorphism and susceptibility to cancers, the results have been conflicting $(7,8)$. However, several studies have consistently implicated the association between the Pro72 variant and poor prognosis among breast and lung cancer (9-12). Thus, while the associations between $p 53$ Arg72Pro polymorphism and cancer risk remain unclear, this polymorphism could modify cancer progression.

In normal cells, p53 is tightly regulated by MDM2 encoded by the human homologue of the mouse double minute 2 gene. MDM2 binds directly to p53, inhibiting its activity and mediating its degradation by ubiquitination (13). While MDM2 maintains p53 at low levels for normal cell growth and development under most physiological conditions, the overexpression of MDM2 attenuates p53 and results in the dysfunction of cell-cycle checkpoint control (13). A previous study showed that an SNP309 ( $\mathrm{T}$ to $\mathrm{G}$ ) in the MDM2 promoter region led to a higher expression level of $M D M 2$ and might accelerate tumor formation among patients with a germline p53 mutation (14). Thereafter, several studies suggested the association of MDM2 SNP309 with cancer risk (15). Recently, it has been reported that the MDM2 SNP309 exhibited an association with the estrogen signaling pathway and environmental stress, such as smoking and viral infection (15), implicating that MDM2 SNP309 may be highly associated with tumor biogenesis.

Transitional cell cancer of the bladder (TCCB) is known to be one of the cancers, which are highly associated with the p53 pathway (2) and many studies have revealed that p53 disruption from mutation and loss of heterozygosity was associated with advanced TCCB $(2,16,17)$. The abnormal expression of MDM2 has shown to be associated with TCC and a subset of advanced TCC without p53 mutation may have MDM2 amplification (18). However, it remains unclear 
whether $p 53$ Arg72Pro and MDM2 SNP309 polymorphism play a critical role in TCCB development. We hypothesized that these polymorphic variants, and their interaction, might be a genetically susceptible factor for the development of TCCB and can influence the clinical course. To test these hypotheses, we conducted a case-control study of a Japanese population and explored the association between TCCB risk and tumor status (stage and grade) and these polymorphisms. Furthermore, we evaluated the association between these polymorphisms and clinical outcome after treatment.

\section{Patients and methods}

Subjects. Between January 1990 and December 2004, 227 patients with newly diagnosed and histologically confirmed bladder cancer and 266 healthy controls were enrolled in this study. Patients who had not received any previous therapy before enrollment were recruited from the Akita University Medical Center and Kyoto University Hospital. Clinical and histopathological information were reviewed from patient medical charts and entered into the access database. The tumor stage was classified into a superficial (pTa, pT1, pTis) and an invasive ( $\geq$ pT2d) group according to the TNM 1997 staging system. The pathological grade was classified into a low grade (G1+G2) and high grade (G3) according to the 1973 WHO criteria (19). Healthy control subjects without a prior history of cancer were recruited from community hospitals in the Akita prefecture. Each of the control subjects were checked with a microscopic examination of urine sediment in order to rule out TCCB. All study participants signed an informed consent and provided a blood sample. Of the 176 patients with superficial TCCB, 87 patients with follow-up information were used for evaluating recurrence-free survival after transurethral resection of the bladder tumor (TUR-Bt). Patients with CIS (pTis) were excluded since CIS shows a wide range of biological aggressiveness and is not potentially superficial. Of the 87 patients, $28(31 \%)$ received intra-vesical chemotherapy after TUR-Bt (24 received epirubicine, 2 mitomicin and 2 BCG). The patients of superficial TCCB were followed up with cystoscopy every 3 months after TUR-Bt. Eighty-six TCC patients who underwent radical cystectomy for invasive and/or high-grade TCCB were used for evaluating diseasespecific survival. Twenty-one patients $(24.4 \%)$ received chemotherapy with cisplatinum-based regimen and 11, 13 and 3 patients received neo-adjuvant, adjuvant and both chemotherapy, respectively. After radical cystectomy, the patients were followed with a CT scan every 3 months for the first 2 years, every 6 months for the next 3 years and every year thereafter until disease progression or death.

Genotyping of MDM2 SNP309 and p53 Arg72Pro polymorphism. DNA was extracted from blood samples collected from patients with TCCB or normal controls using the QIAamp blood Kit (Qiagen, Valencia, CA, USA). The 89-bp fragment containing the $\mathrm{T}$ to $\mathrm{G}$ polymorphic site in the MDM2 intron 1 (MDM2 SNP309) was amplified using specific primers: 5'TTC GGA GGT CTC CGC GGG AGT TCA G and 5'TGC GAT CAT CCG GAC CTC CCG CGT C. Polymerase chain reactions (PCRs) were performed in a $25 \mu 1$ volume containing $20 \mathrm{ng}$ genomic DNA, PCR buffer supplied by a manufacturer, $0.2 \mathrm{mM}$ of each dNTP, $1.5 \mathrm{mM} \mathrm{MgCl}_{2}, 50 \mathrm{pmol}$ of each primer and 1 unit Ampli-Taq DNA polymerase (PE Applied Biosystems, Foster City, CA, USA). After a 12-min initial denaturation step at $95^{\circ} \mathrm{C}, 35$ cycles of PCR consisting of $95^{\circ} \mathrm{C}$ for $30 \mathrm{sec}, 55^{\circ} \mathrm{C}$ for $30 \mathrm{sec}$ and $72^{\circ} \mathrm{C}$ for $60 \mathrm{sec}$ followed by a 7 -min final extension step at $72^{\circ} \mathrm{C}$ were performed in a thermal cycler. Each PCR product was digested overnight with 5 units $\mathrm{Taq}$ I enzyme at $65^{\circ} \mathrm{C}$ (New England Biolabs, Beverly, MA) and electrophoresed on $3.0 \%$ agarose gel. The 89-bp PCR fragment was divided into 64- and 25-bp fragments when the Taq I site was present. The genotype was designated as $\mathrm{T}$ or $\mathrm{G}$ when the Taq I restriction site was present or absent, respectively. Genotyping of the p53 Arg72Pro polymorphism was conducted with the polymerase chain reaction with the confronting two-pair primers (PCRCTPP) method, as described previously (20).

Statistical analysis. All data were analyzed by SPSS software version 11.0 (SPSS, Inc., Chicago, IL). Hardy-Weinberg equilibrium analyses were performed to compare observed and expected genotype frequencies using a Chi-square test (d.f.=1). The odds ratio (OR) and $95 \%$ confidence interval (CI) were calculated as an estimate of the relative risk using a multivariate logistic regression model adjusted for age (continuous variable) and gender. Kaplan-Meier survival curves with the log-rank test were used to evaluate recurrencefree and disease-specific survival among each genotype group. Multivariate analysis using a Cox proportional hazard model was performed to evaluate prognostic factors for the recurrence of superficial TCCB and disease-specific survival of TCCB after radical cystectomy. Results were considered significant at $\mathrm{P}$-value $<0.05$.

\section{Results}

Genotype distributions and cancer risk. Clinicopathological characteristics and the genotype distributions of MDM2 SNP309 and p53 Arg72Pro of the subjects are shown in Table I. The case group comprised of 173 males and 54 females and the control group comprised of 204 males and 62 females. There were no significant differences in the age and gender distributions between the cases and controls. The frequencies of SNP309 G and $p 53$ Pro72 alleles were 0.551 and 0.419 in cases and 0.554 and 0.394 in controls, respectively $(\mathrm{P}=0.86$ and $\mathrm{P}=0.52$, respectively). In the two polymorphisms, the controls showed no deviation from the Hardy-Weinberg equilibrium ( $\mathrm{P}>0.05$, data not shown). There was no significant relationship between the mean age of TCCB onset and the two polymorphic genotypes ( $\mathrm{P}>0.05$, data not shown). Overall, there were no significant associations between each genotype of the two polymorphisms and TCCB risk (Table II). When each group of the SNP309 genotypes was subdivided by the p53 Arg72Pro genotypes (Arg/Arg, Arg/Pro and Pro/Pro), there was no significant association between each group and the TCCB risk (Table II).

Genotype distributions and tumor status (stage and grade). Although we evaluated the effects of MDM2 SNP309 and p53 Arg72Pro polymorphisms on disease status (tumor stage and grade) at the time of diagnosis, there were no statistically 
A

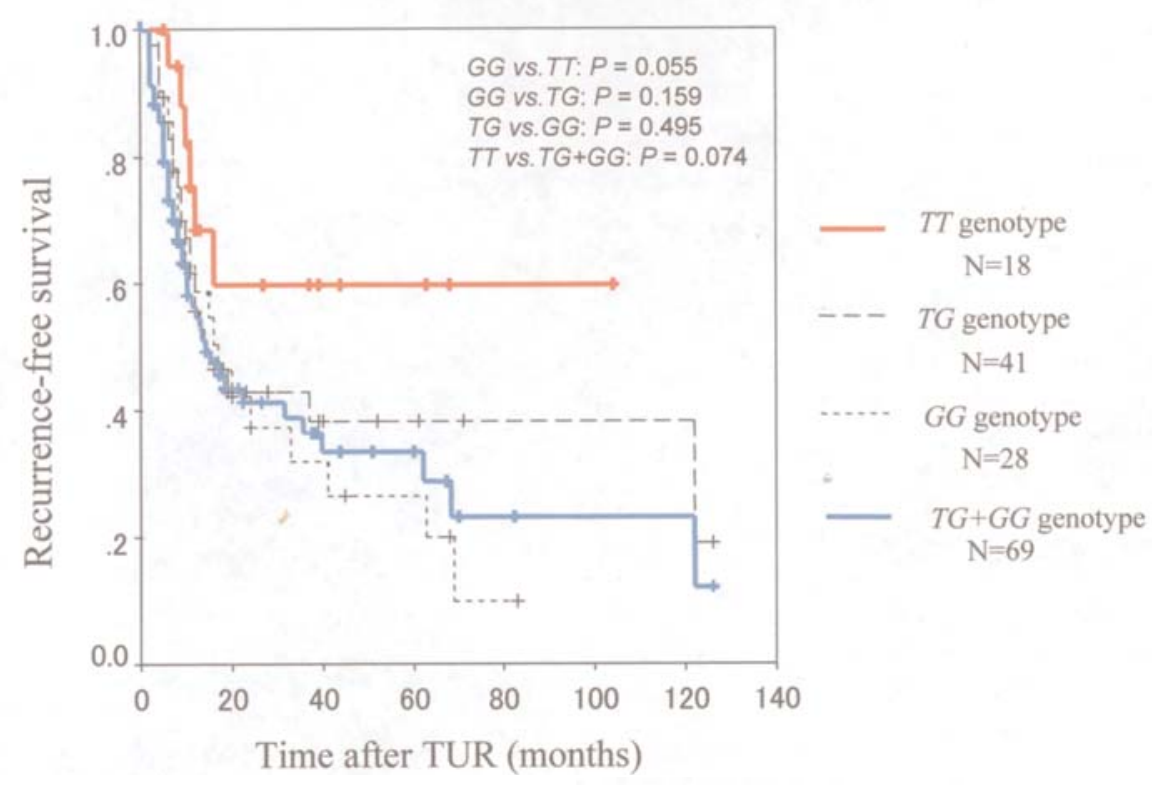

B

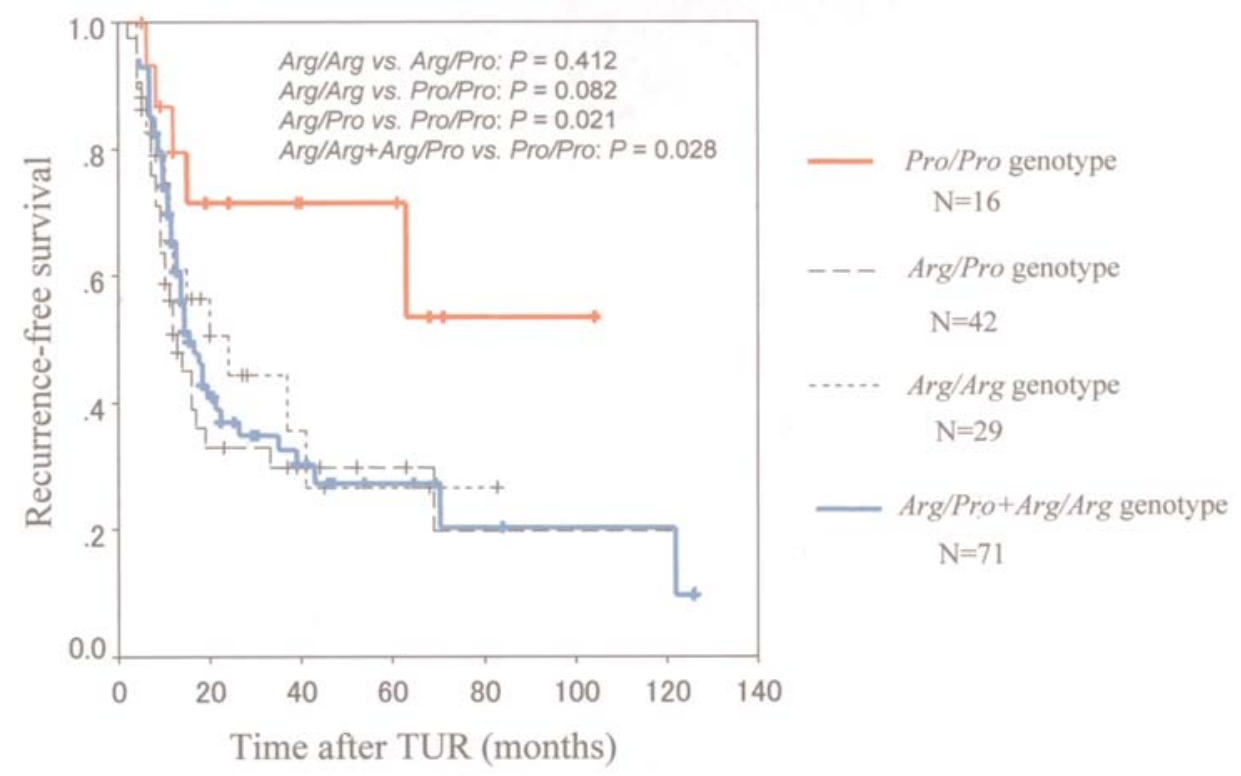

Figure 1. (A) Kaplan-Meier curve of the recurrence-free survival rate in patients with superficial bladder TCC according to the $M D M 2$ polymorphism. (B) Kaplan-Meier curve of the recurrence-free survival rate in patients with superficial bladder TCC according to the $p 53$ Arg72Pro polymorphism.

significant associations between tumor status and each genotype on the two genes (Table II). However, patients with the SNP309 G allele tended to have a higher grade or advanced stage of TCCB with an increased $\mathrm{G}$ allele frequency (Table II). When two polymorphisms were combined, patients with the SNP309 GG and p53 Arg72Pro Pro/Pro genotype showed a 4.47 -fold (95\% CI, 0.83-24.14) increase in risk of high-grade TCCB compared with the SNP309 TT and p53 Arg72Pro Arg/Arg genotype.

Recurrence-free survival according to the MDM2 SNP309 and p53 Arg72Pro genotypes in superficial TCCB. The recurrence-free survival (RFS) rates for the 87 patients with superficial TCCB according to the MDM2 SNP309 or $p 53$ Arg72Pro genotypes were evaluated. Of the 87 patients (72 in pTa and 15 in pT1), $49(56.3 \%)$ had recurrence during the follow-up period (mean: 23.9 months, range: 2-126 months). Although not significant, the patients with the SNP309 TT genotype had a longer RFS compared with the GG or TG genotype ( $\mathrm{P}=0.074$; Fig. 1A). On the $p 53$ Arg72Pro, patients with the Pro/Pro genotype had a significantly lower RFS compared with those with the Arg/Pro or Arg/Arg genotype (Arg/Arg versus Pro/Pro, $\mathrm{P}=0.082$; Arg/Pro versus Pro/Pro, $\mathrm{P}=0.021$; Pro/Pro versus Arg/Arg $+\operatorname{Arg} /$ Pro, $\mathrm{P}=0.028$ by the log-rank test; Fig. 1B). Multivariate analysis using a Cox proportional hazards model showed that the Pro/Pro genotype [hazard ratio (HR), 0.364; 95\% CI, 0.14-0.93] was an independent prognostic factor for recurrence (Table III).

Disease-specific survival according to MDM2 SNP309 and p53 Arg72Pro genotypes in patients who underwent radical cystectomy. Of the 86 patients treated with radical cystectomy, 
Table I. Genotype distribution, demographic characteristics and grade and stage in the 227 patients with TCCB.

\begin{tabular}{|c|c|c|c|c|c|c|c|}
\hline & \multirow[b]{2}{*}{ All } & \multicolumn{3}{|c|}{ MDM2 SNP309 } & \multicolumn{3}{|c|}{ p53 Arg72Pro } \\
\hline & & TT & TG & GG & Arg/Arg & Arg/Pro & Pro/Pro \\
\hline Control group & 266 & $55(20.7)$ & $132(49.6)$ & $79(29.7)$ & $93(34.6)$ & $136(51.1)$ & $38(14.3)$ \\
\hline Case group & 227 & $44(19.4)$ & $116(51.1)$ & $67(29.5)$ & $73(32.2)$ & $118(52.0)$ & $36(15.9)$ \\
\hline \multicolumn{8}{|l|}{ Gender (M/F) } \\
\hline Control group & $204 / 62$ & $35 / 20$ & $106 / 26$ & $63 / 16$ & $72 / 20$ & $101 / 35$ & $31 / 7$ \\
\hline Case group & $173 / 54$ & $36 / 8$ & $80 / 36$ & $57 / 10$ & $57 / 16$ & $92 / 26$ & $24 / 12$ \\
\hline \multicolumn{8}{|l|}{ Mean age \pm SD } \\
\hline Control group & $60.6 \pm 12.2$ & $56.9 \pm 12.5$ & $60.4 \pm 13.5$ & $58.7 \pm 12.4$ & $58.6 \pm 13.7$ & $59.7 \pm 12.7$ & $59.1 \pm 12.6$ \\
\hline Case group & $67.8 \pm 12.6$ & $67.9 \pm 13.2$ & $68.2 \pm 12.3$ & $67.8 \pm 12.5$ & $67.1 \pm 11.9$ & $67.8 \pm 13.5$ & $69.1 \pm 10.7$ \\
\hline \multicolumn{8}{|l|}{ Grade } \\
\hline $1+2$ & 120 & $28(23.3)$ & $60(50.0)$ & $32(26.7)$ & $38(31.7)$ & $66(55.0)$ & $16(13.3)$ \\
\hline 3 & 107 & $16(15.0)$ & $56(52.3)$ & 35 (32.7) & $35(32.7)$ & $52(48.6)$ & $20(18.7)$ \\
\hline \multicolumn{8}{|l|}{ Stage } \\
\hline pTis, pTa, pT1 & 176 & $36(20.5)$ & 89 (50.6) & $51(29.0)$ & $59(33.5)$ & $91(51.7)$ & $26(14.8)$ \\
\hline$\geq \mathrm{pT} 2$ & 51 & $8(15.7)$ & $27(52.9)$ & $16(31.4)$ & $14(27.5)$ & $27(52.9)$ & $10(19.6)$ \\
\hline
\end{tabular}

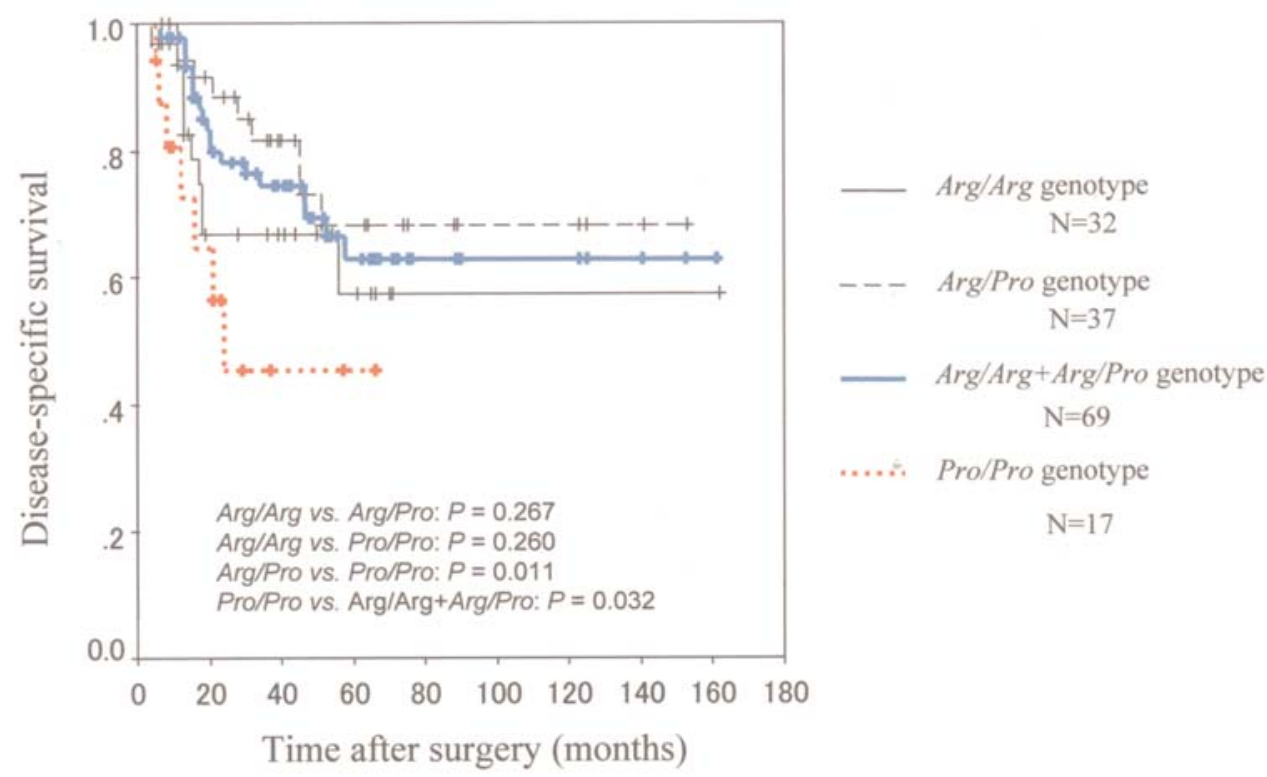

Figure 2. Kaplan-Meier curve for disease-specific survival after radical cystectomy according to the p53 Arg72Pro polymorphism.

$51(59.0 \%)$ had stage $\leq$ pT2 and $35(41.0 \%)$ had $\geq$ pT3; 26 (30.2\%) died during the follow-up period (mean: 36.6 months, range: 4-162 months). On the MDM2 SNP309, there was no significant association between each genotype and diseasespecific survival (TT versus $\mathrm{TG}, \mathrm{P}=0.31$; TT versus $\mathrm{GG}$, $\mathrm{P}=0.96$; TG versus $\mathrm{GG}, \mathrm{P}=0.15$, by the log-rank test). On the p53 Arg72Pro, the patients with the Pro/Pro had a significantly lower survival rate compared with those with the Arg/Pro or those with the Arg/Pro + Arg/Arg (Arg/Pro versus Pro/Pro, $\mathrm{P}=0.019$; Pro/Pro versus Arg/Arg+Arg/Pro, $\mathrm{P}=0.032$ by the log-rank test; Fig. 2). On univariate analysis, the Pro/Pro genotype, stage, lymph node involvement and adjuvant chemotherapy were significant prognostic factors. On multivariate analysis, the Pro/Pro genotype (HR, 2.76; 95\% CI, 1.11-6.84) and lymph node involvement (HR, 4.04; $95 \%$ CI, 1.64-9.95) were independent prognostic factors (Table IV).

\section{Discussion}

In this study, we investigated the impact of MDM2 SNP309 and $p 53$ Arg72Pro polymorphisms on TCCB risk in a Japanese population. However, no significant associations were found even in the combined analyses with the two polymorphisms. For MDM2 SNP309, one study reported that patients with the 
Table II. Risk evaluation of TCCB and tumor status (grade and stage) according to the MDM2 SNP309 and p53 Arg72Pro.

\begin{tabular}{|c|c|c|c|}
\hline & \multicolumn{3}{|c|}{ Adjusted OR (95\% confidence interval) } \\
\hline & $\begin{array}{l}\text { Bladder cancer } \\
\text { against control }\end{array}$ & $\begin{array}{c}\text { Tumor grade } \\
(\mathrm{G} 3 \text { against } \mathrm{G} 1+\mathrm{G} 2)\end{array}$ & $\begin{array}{c}\text { Tumor stage } \\
\text { (invasive against superficial) }\end{array}$ \\
\hline \multicolumn{4}{|c|}{$M D M 2$ SNP309 } \\
\hline TT & 1.00 (Reference) & 1.00 (Reference) & 1.00 (Reference) \\
\hline TG & $1.00(0.59-1.57)$ & $1.57(0.76-3.23)$ & $1.37(0.62-1.63)$ \\
\hline GG & $1.06(0.63-1.85)$ & $1.98(0.90-4.33)$ & $1.41(0.63-1.83)$ \\
\hline \multicolumn{4}{|c|}{ p53 Arg72Pro } \\
\hline Arg/Arg & 1.00 (Reference) & 1.00 (Reference) & 1.00 (Reference) \\
\hline Arg/Pro & $1.08(0.71-1.62)$ & $0.84(0.47-1.51)$ & $0.63(0.24-1.63)$ \\
\hline Pro/Pro & $1.12(0.64-1.98)$ & $1.27(0.57-2.86)$ & $0.79(0.34-1.84)$ \\
\hline \multicolumn{4}{|c|}{ Combined analysis } \\
\hline \multicolumn{4}{|l|}{ TT } \\
\hline Arg/Arg & 1.00 (Reference) & 1.00 (Reference) & 1.00 (Reference) \\
\hline Arg/Pro & $0.91(0.36-2.31)$ & $1.78(0.43-7.33)$ & $0.75(0.14-4.02)$ \\
\hline Pro/Pro & $0.39(0.11-1.47)$ & $1.74(0.21-14.66)$ & $0.91(0.07-11.55)$ \\
\hline \multicolumn{4}{|l|}{ TG } \\
\hline Arg/Arg & $0.57(0.30-1.58)$ & $2.55(0.67-9.70)$ & $0.66(0.13-3.22)$ \\
\hline Arg/Pro & $0.93(0.38-1.92)$ & $2.08(0.59-7.35)$ & $1.37(0.34-5.57)$ \\
\hline Pro/Pro & $1.01(0.36-2.21)$ & $2.91(0.67-12.76)$ & $1.68(0.33-8.55)$ \\
\hline \multicolumn{4}{|l|}{ GG } \\
\hline Arg/Arg & $1.02(0.40-2.58)$ & $3.70(0.90-15.15)$ & $1.35(0.28-6.55)$ \\
\hline Arg/Pro & $0.68(0.29-1.62)$ & $2.11(0.55-8.08)$ & $1.10(0.24-5.03)$ \\
\hline Pro/Pro & $1.11(0.35-3.54)$ & $4.47(0.83-24.14)$ & $1.42(0.22-8.97)$ \\
\hline
\end{tabular}

Table III. Risk evaluation for recurrence-free survival in patients with superficial TCC who underwent TUR-Bt by a Cox proportional hazard model.

\begin{tabular}{|c|c|c|c|c|c|c|}
\hline & \multicolumn{3}{|c|}{ Univariate } & \multicolumn{3}{|c|}{ Multivariate } \\
\hline & HR & $95 \% \mathrm{Cl}$ & $\mathrm{P}$ & HR & $95 \% \mathrm{Cl}$ & $\mathrm{P}$ \\
\hline p53Arg72Pro (Pro/Pro vs. Arg/Arg + Arg/Pro) & 0.38 & $0.15-0.94$ & 0.037 & 0.36 & $0.14-0.93$ & 0.035 \\
\hline Gender (Female vs. Male) & 1.21 & $0.65-2.78$ & 0.055 & & & \\
\hline Age ( $\geq 70$ vs. $<70$ years $)$ & 1.76 & $1.12-2.78$ & 0.014 & 1.61 & $0.89-2.91$ & 0.114 \\
\hline Tumor stage (pT1 vs. pTa) & 0.87 & $0.41-1.86$ & 0.727 & & & \\
\hline Tumor grade (G3 vs. G1+G2) & 1.12 & $0.63-1.99$ & 0.703 & & & \\
\hline Intravesical chemotherapy (Yes vs. No) & 0.66 & $0.34-1.27$ & 0.213 & & & \\
\hline
\end{tabular}

HR, hazard ratio; $95 \% \mathrm{Cl}, 95 \%$ confidence interval.

GG genotype exhibited a 2.68-fold increase in the TCCB risk compared with the TT and TG in a Turkish population (21). However, since the sample size of their study was limited and the allelic frequencies of SNP309 varied according to ethnic background, their results need to be validated by a larger scale study and other ethnic series samples. For p53
Arg72Pro, there have been two studies exploring the association with TCCB risk in a Japanese population $(22,23)$. Although the two studies failed to show a significant association with TCCB risk, one study implied that there is an association between the Pro/Pro genotype and a higher risk of TCC in male smokers (23). However, Soulitizis et al 
Table IV. Risk evaluation for disease-specific survival in patients with radical cystectomy by a Cox proportional hazard model.

\begin{tabular}{|c|c|c|c|c|c|c|}
\hline & \multicolumn{3}{|c|}{ Univariate } & \multicolumn{3}{|c|}{ Multivariate } \\
\hline & HR & $95 \% \mathrm{Cl}$ & $\mathrm{P}$ & HR & $95 \% \mathrm{Cl}$ & $\mathrm{P}$ \\
\hline p53 Arg72Pro (Pro/Pro vs. Arg/Arg + Arg/Pro) & 2.5 & $1.04-6.00$ & 0.04 & 2.76 & $1.11-6.84$ & 0.028 \\
\hline Gender (Female vs. Male) & 1.34 & $0.58-3.09$ & 0.49 & & & \\
\hline Age $(\geq 70$ vs. $<70$ years $)$ & 1.55 & $0.71-3.39$ & 0.269 & & & \\
\hline Tumor stage ( $\geq$ pT3 vs. $<$ pT3) & 4.18 & $1.86-9.48$ & 0.001 & 2.28 & $0.89-5.86$ & 0.087 \\
\hline Lymph node involvement (positive vs. negative) & 6.25 & $2.83-13.77$ & $<0.001$ & 4.04 & $1.64-9.95$ & 0.002 \\
\hline Neo-Adjuvant chemotherapy (Yes vs. No) & 0.69 & $0.20-2.32$ & 0.549 & & & \\
\hline Adjuvant chemotherapy (Yes vs. No) & 3.95 & $1.75-8.91$ & 0.001 & 0.598 & $0.24-1.50$ & 0.274 \\
\hline
\end{tabular}

$\mathrm{HR}$, hazard ratio; $95 \% \mathrm{Cl}, 95 \%$ confidence interval.

reported a significant association between the Arg/Arg genotype and an increased risk of bladder cancer (8).

The analyses between the MDM2 SNP309 and tumor status (stage and grade) showed that the $G$ allele tended to have an increased risk of high-grade TCCB (Table II). These results imply that the $\mathrm{G}$ allele might be associated with an aggressive phenotype. Furthermore, patients with the TT genotype had a higher RFS than those with the TG and GG in superficial TCCB ( $\mathrm{P}=0.074$; Fig. 1A). The SNP309 $\mathrm{G}$ allele might influence the recurrence of superficial TCCB through attenuating p53. On TCCB treated by radical cystectomy, we could not find any association between MDM2 SNP309 and disease-specific survival (DSS). It is well known that p53 function was frequently disrupted in high-grade TCCB because of the mutation and allelic loss of chromosome $17 \mathrm{p}$ $(17 \mathrm{pLOH})(16,17)$. Under these conditions, SNP309 might not have an effect on p53. Sanchez-Carbayo et al (24) reported that invasive TCCB patients with the TT genotype had a poorer prognosis than those with the TG and GG since patients with the TT might lead to a greater chance of having p53 mutation. However, when they evaluated the p53 mutation with the clinical outcome in patients with the TG or GG, p53 mutation exhibited a significantly poorer prognostic effect, suggesting that the p53 status affects the clinical outcome regardless of SNP309. Although their results were obtained from a series with a different background from ours, our results and theirs both indicated that SNP309 was unlikely to have an affect on the clinical outcome in the aggressive TCCB.

On the $p 53$ Arg72Pro, we obtained contradictory results on the outcome between superficial TCCB and aggressive TCCB treated with radical cystectomy. On superficial TCCB, the Pro/Pro genotype was a favorable prognostic factor, whereas on TCCB treated with radical cystectomy, the Pro/Pro genotype influenced DSS as an unfavorable factor. Previous studies have proposed that TCCB had the two distinct molecular pathways between the superficial and invasive type (17). Superficial TCCB have an activated ras-MAPK signal transduction pathway through FGFR3 gene mutation, whereas invasive TCCB may be initiated by disrupted p53 function through mutation $(2,16,17)$. Our results indicated that the $p 53$
Arg72Pro might function differently between superficial and invasive TCCB. The Arg72 allele has been shown to be more efficient in apoptosis induction than the Pro72 allele through several mechanisms (1,3-6). However, these effects have shown to vary among different tissues $(1,6)$. Besides apoptosis, p53 regulates the various DNA-repair processes independent of its apoptotic function. Siddique and Sabapathy (25) reported that the Pro/Pro genotype has a significantly higher DNA-repair capacity than the Arg/Arg. They showed that DNA-repair target genes, such as gadd45, p48 and p53R2, were more efficiently activated in cells with the Pro72 allele than those with Arg72. Moreover, they showed that cells expressing the Pro72 allele had a reduced genomic instability. In superficial TCCB, the more efficient DNA-repair function from the Pro72 allele might have an affect on inhibiting subsequent genetic alterations and tumor recurrence and progression.

In TCCB treated with radical cystectomy, the Pro/Pro genotype was an independent prognostic factor. This finding was consistent with previous reports from other cancers, such as lung (9) and breast cancer (10-12). Although the mechanism remains unclear, it might be due to the reduced apoptotic efficacy associated with the Pro 72 allele. Since p53 mutation with $17 \mathrm{pLOH}$ has often been found in invasive TCCB (16), the Pro72 allele with p53 mutation may have a more reduced apoptotic efficacy than the Arg allele with p53 mutation in TCCB. Furthermore, several studies suggested that $17 \mathrm{pLOH}$ frequently occurred in the Pro72 allele in certain cancers $(9,26)$. Considering these findings, LOH might occur in the Pro72 allele preferentially compared to the Arg72 allele in invasive TCCB. In other words, p53 with the Pro/Pro genotype might be more prone to lose its function through 17p LOH more than those with the Arg/Pro or Arg/Arg and this genetic event might lead to a poor prognosis. Furthermore, it has been suggested that the different sensitivity to chemotherapy and radiation according to the p53 Arg72Pro could influence a treatment outcome, whereas poor responses to drugs derived from the Pro72 allele have been shown only in tumors with wild-type p53 $(1,27,28)$. Therefore, a subset of aggressive TCCB with wild-type p53 might have been 
influenced by the lower sensitivity associated with the Pro72 allele. In conclusion, there were no significant associations between MDM2 SNP309 and p53 Arg72Pro polymorphisms and TCCB risk in a Japanese population. While the $p 53$ Arg72Pro Pro/Pro genotype had a significantly worse impact on the outcome after radical cystectomy, it showed a protective effect against recurrence in superficial TCCB. These results may reflect marked differences in the genetic background between superficial and invasive TCCB. To validate these findings, a larger scale study with detailed genetic profiling will be needed.

\section{Acknowledgements}

We thank Ms. Yukiko Sugiyama and Ms. Yoko Mitobe for their assistance with DNA preparation and genotyping. This study was supported by grants-in-aid for Scientific Research from the Japan Society for Promotion of Science, Japan (\#19390411,\#19591832, \#19591833 and \#19659406).

\section{References}

1. Soussi T and Wiman KG: Shaping genetic alterations in human cancer: the p53 mutation paradigm. Cancer Cell 12: 303-312, 2007.

2. Smith ND, Rubenstein JN, Eggener SR and Kozlowski JM: The p53 tumor suppressor gene and nuclear protein: basic science review and relevance in the management of bladder cancer. J Urol 169: 1219-1228, 2003.

3. Thomas M, Kalita A, Labrecque S, Pim D, Banks L and Matlashewski G: Two polymorphic variants of wild-type p53 differ biochemically and biologically. Mol Cell Biol 19: 1092-1100, 1999.

4. Dumont P, Leu JI, Della Pietra AC III, George DL and Murphy M: The Arg72Pro polymorphic variants of p53 have markedly different apoptotic potential. Nat Genet 33: 357-365, 2003.

5. Bergamaschi D, Gasco M, Hiller L, Sullivan A, Shed N, Trigiante G, Yulung I, Merlano M, Numico G, Comino A, Attard M, Reelfs O, Gusterson B, Bell AK, Geath V, Tavassoli M, Farrell PJ, Smith P, Lu X and Crook T: p53 polymorphisms influences response in cancer chemotherapy via modulation of p73-dependent apoptosis. Cancer Cell 3: 387-402, 2003.

6. Bergamaschi D, Samuels Y, Sullivan A, Zvelebil M, Breyssens H, Bisso A, Del Sal G, Syed N, Smith P, Gasco M, Crook T and Lu X: iASPP preferentially binds p53 proline-rich region and modulates apoptotic function of Arg72Pro polymorphic p53. Nat Genet 38: 1133-1141, 2006.

7. Pietsch EC, Humbey O and Murphy ME: Polymorphisms in the p53 pathway. Oncogene 25: 1602-1611, 2006.

8. Soulitzis N, Sourvinos G, Dokianakis DN and Spandidos DA: p53 codon 72 polymorphism and its association with bladder cancer. Cancer Lett 179: 175-183, 2002.

9. Nelson HH, Wilkojmen M, Marsit CJ and Kelsey KT: TP53 mutation, allelism and survival in non-small cell lung cancer. Carcinogenesis 26: 1770-1773, 2005.

10. Tommiska J, Eerola H, Heinonen M, Salonen L, Kaare M, Tallila J, Ristimäki A, von Smitten K, Aittomäki K, Heikkilä P, Blomqvist $\mathrm{C}$ and Nevanlinna $\mathrm{H}$ : Breast cancer patients with p53 pro72 homozygous genotype have a poorer survival. Clin Cancer Res 11: 5098-5103, 2005.

11. Xu Y, Yao L, Ouyang T, Li J, Wang T, Fan Z, Lin B, Lu Y and Xie Y: p53 Arg72Pro polymorphism predicts the pathologic response to neoadjuvant chemotherapy in patients with breast cancer. Clin Cancer Res 11: 7328-7333, 2005.
12. Toyama T, Zhang Z, Nishio M, Hamaguchi M, Kondo N, Iwase H, Iwata H, Takahashi S, Yamashita H and Fujii Y: Association of TP53 Aro72Pro polymorphism and the outcome of adjuvant therapy in breast cancer patients. Breast Cancer Res 9: R34, 2007.

13. Marchenko ND and Moll UM: The role of ubiquitination in the direct mitochondrial death program of p53. Cell Cycle 6: 1718-1723, 2007.

14. Bond GL, Hu W, Bond EE, Robins H, Lutzker SG, Arva NC, Bargonetti J, Bartel F, Taubert H, Wuerl P, Onel K, Yip L, Hwang SJ, Strong LC, Lozano G and Levine AJ: A single nucleotide polymorphism in the MDM2 promoter attenuates the p53 tumor suppressor pathway and accelerates tumor formation in humans. Cell 119: 591-602, 2004.

15. Bond GL and Levine AJ: A single nucleotide polymorphism in the p53 pathway interacts with gender, environmental stresses and tumor genetics to influence cancer in humans. Oncogene 27: 1317-1323, 2007.

16. Sugano $\mathrm{K}$ and Kakizoe T: Genetic alterations in bladder cancer and their clinical applications in molecular tumor staging. Nat Clin Pract Urol 41: 642-652, 2006.

17. Mitra AP, Datar TH and Cote RJ: Molecular pathways in invasive bladder cancer: new insights into mechanisms, progression, and target identification. J Clin Oncol 35: 5552-5564, 2006.

18. Habuchi T, Kinoshita H, Yamada H, Kakehi Y, Ogawa O, Wu WJ, Takahashi R, Sugiyama T and Yoshida O: Oncogene amplification in urothelial cancers with p53 gene mutation or MDM2 amplification. J Natl Cancer Inst 86: 1331-1335, 1994.

19. Mostofi FK, Sobin LH and Troloni H: Histological typing of urinary bladder tumors. In: International Histological Classification of Tumors, no. 10. World Health Organization, Geneva, pp15-34, 1973.

20. Huang XE, Hamajima N, Katsuda N, Matcuo K, Hirose K, Mizutani M, Iwata H, Miura S, Xiang J, Tokudome S and Tajima K: Association of p53 codon Arg72Pro and p73G4C14to-A414T at exon 2 genetic polymorphisms with the risk of Japanese breast cancer. Breast Cancer 10: 307-311, 2003.

21. Onat OE, Tez M, Ozcelik T and Toruner GA: MDM2 T309G polymorphisms is associated with bladder cancer. Anticancer Res 26: 3473-3475, 2006

22. Wu WJ, Kakehi Y, Habuchi T, Kinoshita H, Ogawa O, Terachi T, Huang $\mathrm{CH}$, Chiang $\mathrm{CP}$ and Yoshida $\mathrm{O}$ : Allelic frequency of p53 gene ARG72PRO polymorphism in urologic cancers. Jpn J Cancer Res 86: 730-736, 1995.

23. Kuroda Y, Tsukino H, Nakao H, Imai H and Katoh T: p53 ARG72PRO polymorphism and urothelial cancer risk. Cancer Lett 189: 77-83, 2003.

24. Sanchez-Carbayo M, Socci ND, Kirchoff T, Erill N, Offit K, Bocher BH and Cordon-Cardo $\mathrm{C}$ : A polymorphism in HDM2 (SNP309) associates with early onset in superficial tumors, TP53 mutations, and poor outcome in invasive bladder cancer. Clin Cancer Res 13: 3215-3219, 2007.

25. Siddique $M$ and Sabapathy K: Trp53-dependent DNA-repair is affected by the ARG72PRO polymorphism. Oncogene 25: 3489-3500, 2006

26. Furihata M, Takeuchi T, Matsumoto M, Kurabayashi A, Ohtsuki Y, Terao N, Kuwahara M and Shuin T: p53 mutation arising in Arg72 allele in the tumorigenesis and development of carcinoma of the urinary tract. Clin Cancer Res 8: 1192-1195, 2002.

27. Sullivan A, Syed N, Gasco M, Bergamschi D, Trigiante G, Attard M, Hiller L, Farrel PJ, Smith P, Lu X and Crook T: Polymorphism in wild-type p53 modulates response to chemotherapy in vitro and in vivo. Oncogene 23: 3328-3337, 2004.

28. Song $\mathrm{H}$ and $\mathrm{Xu} \mathrm{Y}$ : Gain of function of p53 cancer mutants in disrupting critical DNA damage response pathways. Cell Cycle 6: 1570-1573, 2007. 